\title{
Recursos ambientais, tipos de brinquedos \\ e práticas familiares que potencializam o desenvolvimento cognitivo infantil
}

Sabrina da Conceição Guedes² Rosane Luzia de Souza Morais ${ }^{3}$ (B) Juliana Nogueira Pontes Nobre ${ }^{4}$ (1) Juliana Nunes Santos ${ }^{1}$ (C)

\section{Descritores}

Desenvolvimento Infantil Jogos e Brinquedos Relação Familiar Pré-escolar Poder Familiar

\section{Keywords}

Child Development Play and Playthings Family Relations

Preschool Parenting

Endereço para correspondência: Sabrina da Conceição Guedes Programa de Pós-graduação em Reabilitação e Desempenho Funcional, Universidade Federal do Vale do Jequitinhonha e Mucuri - UFVJM Rodovia MGT 367, Km 583, 5000, Alto da Jacuba, Diamantina (MG), Brasil, CEP: 39100-000. E-mail: sabrinaguedes.ufvjm@gmail. com

Recebido em: Maio 27, 2019

\section{RESUMO}

Objetivo: Identificar fatores associados ao desenvolvimento cognitivo de crianças de 24 a 42 meses de idade, assim como caracterizar a disponibilidade de brinquedos e recursos presentes no ambiente familiar e as práticas parentais que sinalizam estabilidade familiar. Método: Estudo transversal analítico realizado com crianças regularmente matriculadas em instituições de ensino públicas e privadas de uma cidade de médio porte. O desenvolvimento cognitivo foi avaliado por meio do teste Bayley e a qualidade do ambiente que vive a criança foi avaliada utilizando-se o Inventário de Recursos do Ambiente Familiar- RAF adaptado. Para conhecimento do nível econômico das famílias utilizou-se o Critério de Classificação Econômica Brasil 2015 (CCEB). As crianças foram alocados em dois grupos com base no resultado do teste cognitivo e comparadas quanto às atividades e rotinas da família e recursos presentes no domicílio. Resultados: Das 104 crianças avaliadas, $72 \%$ são matriculadas na rede de ensino público, $69 \%$ pertencentes as classes econômicas C e D. De acordo com o desenvolvimento cognitivo, $55 \%$ obtiveram desenvolvimento acima da média aritmética. $\mathrm{Na}$ análise bivariada observou-se que os passeios e viagens realizados pela criança, maior escolaridade materna e paterna, vínculo com creches particulares, assim como a maior disponibilidade de recursos e níveis econômicos maiores foram relacionados a melhores escores cognitivos. Os dois últimos fatores permaneceram como preditores do desenvolvimento cognitivo na análise de regressão logística binária. Conclusão: Maior disponibilidade de recursos no ambiente familiar e níveis econômicos foram positivamente associados ao desenvolvimento cognitivo.

\begin{abstract}
Purpose: To identify the factors associated with the cognitive development of children from 24 to 42 months of age, as well as to characterize the availability of toys and resources present in the family environment, and the parental practices that signal family stability. Methods: Cross-sectional analytical study conducted with children regularly enrolled in public and private educational institutions of a medium-sized city. Cognitive development was assessed by means of the Bayley test and the quality of the environment was evaluated using the Adapted Family Environment Resource Inventory (FERI). The children were allocated into two groups based on the cognitive test result and compared regarding activities performed at home; reports of outings and trips in the last year; presence of regular scheduled activities; activities developed with parents; toys the child has or has had; presence of newspapers, magazines, and books at home; the person responsible for monitoring the child during day-care; and routines of the child and family. Results: Of the 104 children evaluated, $72 \%$ were enrolled in the public education network and $69 \%$ belonged to economic classes C and D. Regarding cognitive development, $55 \%$ had above-average development. In the bivariate analysis, it was observed that greater availability of toys and materials for the child and higher economic levels were related to better scores on the cognitive development test. These remained as predictors of cognitive development in binary logistic regression analysis. Conclusion: Greater availability of resources in the family environment and economic levels were positively associated with cognitive development in children.
\end{abstract}

Trabalho realizado no Departamento de Fisioterapia, Universidade Federal do Vale do Jequitinhonha e Mucuri - UFVJM - Diamantina (MG), Brasil.

Departamento de Fisioterapia, Universidade Federal do Vale do Jequitinhonha e Mucuri - UFVJM - Diamantina (MG), Brasil

${ }^{2}$ Programa de Pós-graduação em Reabilitação e Desempenho Funcional, Universidade Federal do Vale do Jequitinhonha e Mucuri - UFVJM - Diamantina (MG), Brasil.

${ }^{3}$ Mestrado Profissional em Sociedade, Saúde e Meio Ambiente, Universidade Federal dos Vales do Jequitinhonha e Mucuri - UFVJM - Diamantina (MG), Brasil.

${ }^{4}$ Programa de Pós-graduação em Ciências Fonoaudiológicas, Faculdade de Medicina, Universidade Federal de Minas Gerais - UFMG - Belo Horizonte (MG), Brasil.

Fonte de financiamento: nada a declarar.

Conflito de interesses: nada a declarar. 


\section{INTRODUÇÃO}

O desenvolvimento é definido como um processo de mudanças nas estruturas físicas e neurológicas, cognitivas e comportamentais, que emergem de maneira ordenada e são relativamente duradouras ${ }^{(1)}$. $\mathrm{O}$ desenvolvimento infantil é multifatorial, resultado da combinação de fatores biológicos, ambientais, familiares e sociais, de risco ou de proteção, que culminam em um desenvolvimento único e peculiar ${ }^{(2)}$, resultando em uma progressão ordenada do desenvolvimento motor, cognitivo, linguagem, socioemocional e autorregulação ${ }^{(3)}$. Segundo a Organização Mundial da Saúde (OMS), essa fase é extremamente sensível para o desenvolvimento do ser humano, momento de formação de toda a sua estrutura emocional e afetiva, e desenvolvimento de áreas fundamentais do cérebro relacionadas à personalidade, ao caráter e à capacidade de aprendizado ${ }^{(4)}$. Os primeiros três anos de vida, especialmente, denominados como primeira infância ou mais recentemente, primeríssima infância $^{(5)}$ são ainda mais fundamentais para o desenvolvimento da criança, pois nesse momento, as mesmas são favorecidas pela plasticidade cerebral, o chamado período sensível do desenvolvimento cerebral ${ }^{(6)}$.

O desenvolvimento, quando ocorre de forma satisfatória, principalmente nos primeiros anos de vida contribui para o alcance das potencialidades do sujeito, para tornar-se um cidadão mais resolvido, apto a enfrentar as adversidades que a vida oferece, podendo interferir assim nas disparidades sociais e econômicas da nossa sociedade ${ }^{(7)}$. Múltiplos fatores influenciam a aquisição de competências, incluindo saúde, nutrição, segurança, cuidado e aprendizagem precoce; esses domínios interagem uns com os outros e podem ser mutuamente reforçados por meio do processo de desenvolvimento natural ${ }^{(3)}$.

No entanto, esse desenvolvimento natural das crianças pode estar em "risco" em decorrência de alguns fatores, sejam eles fatores biológicos (por exemplo, desnutrição, infecções, anemia, prematuridade), fatores psicossociais (por exemplo, estimulação cognitiva inadequada, exposição à violência, assistência familiar precária ou ausente) e fatores de sociodemográficos (por exemplo: pobreza) ${ }^{(3)}$. A existência de um ou mais fatores podem caracterizar adversidades no percurso de vida da criança e evidências científicas relacionam variações ambientais adversas durante o início da vida (do período fetal à infância) a mudanças de longo prazo no volume, microestrutura e conectividade cerebral, sendo que o acúmulo de adversidades, desde antes da concepção, pré-natal e início da vida, pode atrapalhar o desenvolvimento do cérebro ${ }^{(3)}$, podendo aumentar o risco de crianças pequenas manifestarem problemas de desenvolvimento ${ }^{(8)}$. Destarte, é importante a detecção precoce desses fatores de risco que podem influenciar o desenvolvimento infantil ${ }^{(8)}$.

O baixo nível socioeconômico e a pobreza são considerados fatores de risco para o desenvolvimento infantil, não somente porque limitam a aquisição de bens, mas também influenciam o acesso aos conteúdos educacionais. Em casos de pobreza extrema, o indivíduo se torna vulnerável, isto é, com maior chances de não alcançar seu máximo potencial de desenvolvimento por ser privado de estímulos e experiências propulsoras do desenvolvimento e aprendizado ${ }^{(3)}$. A pobreza e as experiências adversas na infância têm efeitos sobre o desenvolvimento do cérebro e da cognição a longo prazo, ao passo que a condição socioeconômica desfavorável está associada ao déficit da linguagem e cognição aos três anos de idade e a problemas ainda piores à idade de cinco anos ${ }^{(3)}$.

O ambiente familiar desempenha um importante papel na mediação do bem-estar de criança expostas num ambiente com baixo nível socioeconômico. Em casos de atrasos no desenvolvimento, práticas parentais positivas e variedade de outros fatores como, saúde mental dos pais e ausência de violência no ambiente familiar assumem um papel protetor particularmente significativo na mediação parcial dos efeitos negativos da vulnerabilidade socioeconômica ${ }^{(9,10)}$. A família é o primeiro meio onde a criança é inserida, e a mesma precisa ser mediadora entre a criança e a sociedade, sendo vista como espaço de socialização infantil. A estabilidade familiar, sinalizada por relações interpessoais confiáveis e estáveis, rotinas regulares e organização do ambiente doméstico, tem sido identificada como um fator de proteção contra problemas de comportamento. Em contrapartida, um ambiente doméstico caótico, com grande instabilidade diária, desordem e falta de regularidade temporal, constitui fator de risco para a intensificação de problemas $^{(11)}$.

Estudos demonstraram que a variável de maior impacto sobre o desenvolvimento infantil é a estimulação no ambiente familiar ${ }^{(12)}$, com influências positivas no desempenho cognitivo ${ }^{(12)}$, neuropsicomotor ${ }^{(13)} \mathrm{e}$ da linguagem ${ }^{(14)}$. As dificuldades familiares estão associadas significativamente ao aumento de risco de problemas de comportamento infantil e atraso no desenvolvimento. $\mathrm{O}$ ambiente familiar e suas relações constituem a base para promover o desenvolvimento afetivo-social e cognitivo da criança. Assim, a família pode atuar como estimuladora e protetora desse processo ao fornecer suporte parental, experiências variadas e de qualidade e ao proporcionar a interação com adultos e crianças por meio de rotinas e reuniões regulares da família que sinalizam algum grau de estabilidade familiar ${ }^{(15)}$. No entanto, também pode constituir fator de risco, ao privar a criança de um ambiente familiar estruturado e com estímulos adequados ${ }^{(12)}$.

Dentro do ambiente familiar as brincadeiras devem ser rotineiras para as crianças, especialmente, quando realizadas com os familiares ${ }^{(16)}$. Brincar com brinquedos de várias maneiras reflete a exploração e a descoberta de suas possibilidades. Isso pode aumentar as oportunidades para o desenvolvimento da criatividade, imaginação, e desenvolvimento de habilidades ${ }^{(17)}$. $\mathrm{O}$ tipo de brinquedo pode melhorar o desenvolvimento das sensações, movimentos finos, e potencializar a capacidade cognitiva da criança, como pode também ser uma forma de treinamento para sua capacidade de pensar, raciocinar, ponderar riscos, de modo que ele possa enfrentar um ambiente em mudança mais facilmente ${ }^{(16)}$. Torna-se necessário o entendimento de quais recursos ou brinquedos potencializam o desenvolvimento cognitivo infantil, a fim de ofertá-los as crianças em suas rotinas diárias, para estimulá-las ou evitar possíveis atrasos.

O presente estudo teve como objetivo identificar fatores associados ao desenvolvimento cognitivo de crianças de 24 a 42 meses de idade, assim como caracterizar a disponibilidade de brinquedos e recursos presentes no ambiente familiar e as práticas parentais que sinalizam estabilidade familiar. 


\section{MÉTODO}

Trata-se de um estudo transversal analítico, realizado no período de novembro de 2016 a fevereiro de 2017.

O presente estudo é parte de um projeto de pesquisa mais amplo aprovado pelo Comitê de Ética em Pesquisa institucional sobre o número CAAE - 55459916.0.0000.5108.

\section{Amostra}

A amostra foi calculada a partir de um total de 576 crianças, regularmente matriculadas, nas nove unidades pré-escolares do município de Diamantina (MG). Para o cálculo estimou-se prevalência do desenvolvimento cognitivo abaixo da média igual a $28,3 \%{ }^{(18)}$ com precisão desejada de $5 \%$, intervalo de confiança de $95 \%$, resultando em 86 crianças. A esse valor acrescentou-se $20 \%$ de perdas, totalizando 103 crianças.

Foram elegíveis para o estudo crianças de desenvolvimento típico, com idade entre 24 a 42 meses, por ser a idade média que as crianças ingressam nas escolas, matriculadas em creches públicas e particulares do município, devidamente autorizadas pelos pais. Foram excluídos aqueles cujos pais não assinaram o TCLE, as crianças com paralisia cerebral ou síndromes que afetam a cognição e os aspectos linguísticos, crianças diagnosticadas com autismo, doenças neurodegenerativas e crianças com perda de audição, sendo estas informações relatadas pelos pais ou pela escola.

Participaram do estudo 104 crianças, com idade entre 24 a 42 meses, com mediana de 35 meses, e seus respectivos familiares. Das crianças, 53 (51\%) são do sexo masculino, e 51 (49\%) feminino. Quanto a instituição de ensino, 75 (72\%) são matriculadas no ensino público e 29 (28\%) na rede particular. Quanto à classificação socioeconômica, as famílias são assim distribuídas: sete famílias pertencem a categoria A; 24 a categoria B; 21 a categoria $C 1 ; 27$ a categoria $C 2$; e 24 a categoria D-E.

\section{Instrumentos}

Para avaliar o desenvolvimento infantil foi utilizado o instrumento Bayley III, terceira edição, padrão-ouro, tradicionalmente conhecida e bastante utilizada na prática clínica e em pesquisas científicas para a avaliação do desenvolvimento infantil ${ }^{(19)}$. O teste tem como principal objetivo identificar crianças com atraso no desenvolvimento na faixa etária de um a 42 meses nos domínios: cognitivo, linguagem (expressiva e receptiva), motor (fino e grosso), socioemocional e comportamento adaptativo. Para o presente estudo, foi utilizada apenas a escala cognitiva e de acordo com o manual ${ }^{(20)}$, foi utilizado o escore balanceado, onde dez é a pontuação média com desvio-padrão de \pm três pontos.

A qualidade do ambiente que vive a criança foi avaliada utilizando-se o Inventário de Recursos do Ambiente Familiar$\mathrm{RAF}^{(15)}$, com adaptações, ou seja, foram retirados do instrumento itens direcionados para crianças fora da faixa etária do estudo ${ }^{(13)}$. O RAF tem por objetivo avaliar os recursos do ambiente familiar em três domínios: 1) Recursos que promovem processos proximais: compreende participação em experiências estimuladoras para o desenvolvimento, como passeios e viagens, oportunidades de interação com os pais; disponibilidade de brinquedos e materiais que apresentam desafio ao pensar; disponibilidade de livros, jornais e revistas, uso adequado do tempo livre; 2) Atividades que sinalizam estabilidade na vida familiar: como rotinas e reuniões regulares da família e cooperação da criança em tarefas domésticas; 3) Práticas parentais que promovem o vínculo família-escola: envolvimento direto dos pais na vida escolar, como participação nas reuniões ${ }^{(15)}$. Para análise foram considerados o somatório de pontos (pontuação bruta) e a fórmula pontuação bruta/ pontuação máxima do tópico para obtenção da pontuação relativa, sendo que quanto maior é a pontuação adquirida no RAF maior é a qualidade do ambiente.

Neste estudo, para conhecimento do nível econômico das famílias utilizou-se o Critério de Classificação Econômica Brasil 2015 (CCEB) da ABEP. O instrumento atribui uma pontuação por item baseada em três variáveis: poder de compra da família, escolaridade da pessoa de referência e serviços públicos que a família possui em seu domicílio. Em seguida é gerado um escore geral que varia de 0 a 100 pontos que, por fim, classifica o nível socioeconômico da família em uma escala ordinal crescente que varia de $\mathrm{E}$ a $\mathrm{A} 1^{(21)}$. Para fins de análise as classes foram categorizadas em dois grupos: nível socioeconômico alto "A, $\mathrm{B}, \mathrm{C} 1$ ” e nível socioeconômico baixo em "C2, D, E”(22).

\section{Procedimentos}

Inicialmente foi obtida a autorização da Secretaria Municipal do para a execução da pesquisa. Foi realizado um contato prévio com os diretores (a) das creches particulares e públicas de Diamantina para esclarecimentos sobre a pesquisa e autorização. Em seguida o termo de consentimento livre e esclarecido (TCLE) e o questionário econômico foram enviados nas mochilas de todas as crianças com idade compatível ao estudo, para o preenchimento pelos pais. No segundo momento, foi realizado plantão nas instituições de ensino nos horários de entrada e saída das crianças para realização da entrevista semi-estruturada com os pais, utilizando o questionário padronizado RAF.

A aplicação da Escala Bayley de Desenvolvimento Infantil se deu após o treinamento dos aplicadores. O treinamento foi conduzido por uma fisioterapeuta, capacitada na aplicação do teste e ao seu término procedeu-se a avaliação da confiabilidade entre os examinadores e o Índice de Correlação Intraclasse (ICC) obtida foi de $0,95 \%$. A escala cognitiva do Bayley III foi aplicada por 04 examinadores em escala de revezamento. A aplicação se deu em sala disponibilizada pela escola, no dia e horário agendados pela coordenação da instituição de ensino sempre realizada por dois examinadores.

Para fins de análise o resultado do teste cognitivo do Bayley foi dicotomizado pela pontuação dez no escore balanceado, por ser essa a pontuação média do teste, cujo desvio padrão é \pm três pontos. As crianças foram alocadas em dois grupos com base no resultado do teste cognitivo do Bayley, a saber: grupo abaixo da média e grupo médio ou superior.

\section{Análises de dados}

Os dados coletados foram transferidos inicialmente para o Statistical Packege for the Social Sciences (SPSS) versão 19.0, quando foi realizada análise exploratória do banco de dados e análises descritivas dos participantes e das variáveis do estudo.

O Teste Shapiro-Wilk foi empregado para análise da distribuição normal das variáveis contínuas e os dados estão apresentados em média \pm DP. As variáveis de caracterização da amostra foram comparadas entre os grupos pelo teste Mann-Whitney. A 
comparação da distribuição das variáveis categóricas entre os grupos foi realizada pelo teste qui-quadrado e estão apresentadas por número absoluto e frequência relativa.

Para análise das variáveis escolaridade materna e paterna, as respostas foram dicotomizadas em duas categorias de acordo com a Lei de Diretrizes e Bases da Educação Nacional (LDB) ${ }^{(23)}$ : Educação Básica e Educação Superior.

Para análise da qualidade do ambiente familiar considerou-se os resultados de cada questão do RAF separadamente, a saber: 1) Atividades realizadas pela criança em casa; 2) Passeios realizados pela criança; 3) Atividades programadas que a criança realiza regularmente; 4) Atividades desenvolvidas com os pais; 5) Brinquedos que a criança tem ou já teve; 6) Presença de jornais ou revistas no domicílio; 7) Presença de livros em casa; 8) Quem acompanha a criança nos afazeres da creche; 9) Rotina da criança; 10) Rotina da família. Os itens avaliados na questão 4, brinquedos que a criança tem ou já teve, foram analisados separadamente segundo o desenvolvimento cognitivo dos infantes. No entanto, para a análise multivariada considerou-se somente o resultado da questão quatro, formado pelo somatório dos recursos da criança.

No modelo de regressão linear binária hierárquica foram consideradas as variáveis que tiveram um $\mathrm{p}<0,10$ na análise bivariada e permaneceram no modelo apenas aquelas com $\mathrm{p}<0,05$. Interações entre as variáveis do modelo final foram examinadas e foram retiradas do modelo final as variáveis "escolaridade materna" e "tipo de creche". A qualidade do modelo foi avaliada pelo método Hosmer-Lemeshow e pela medida de ajuste - 2 log likelihood (-2LL). A análise de resíduos no modelo final foi realizada para detecção de outliers significativos.

\section{RESULTADOS}

De acordo com o desenvolvimento cognitivo, 57 (55\%) crianças obtiveram desenvolvimento acima da média e 47 (45\%) abaixo da média. De acordo com os resultados do Bayley III 7 (6,7\%) foram classificadas com o desenvolvimento cognitivo muito superior, quatro $(3,8 \%)$ superior, 14 (13,5\%) médio alto, $32(30,8 \%)$ com desenvolvimento médio, 42 (40,4\%) médio baixo e cinco $(4,8 \%)$ limítrofe.

$\mathrm{Na}$ Tabela 1 podem ser visualizadas as características socioeconômicas da população estudada e sua relação com o desenvolvimento cognitivo. Foi possível observar que as variáveis nível econômico inferior $(\mathrm{C} 2, \mathrm{D}$ e E), tipo de creche pública, menor escolaridade materna e paterna foram associadas ao desenvolvimento cognitivo abaixo da média das crianças avaliadas.

Na Figura 1 estão descritas as características do ambiente familiar e práticas parentais.

Das atividades realizadas dentro do domicílio pela criança, as mais relatadas pelos pais ou responsáveis foi "assistir TV" $\mathrm{e}$ "brincar em casa" (92,3\%). Das atividades realizadas juntamente com os pais "o brincar" (94,2\%), "assistir programas de TV" (76,9\%) e "ler livros" (71,2\%) foram as de maior ocorrência. Em relação aos recursos das crianças os brinquedos de andar $(83,7 \%)$, bola, pipa, bola de gude $(77,9 \%)$ e brinquedos com cores e formas $(76 \%)$ foram os mais citados.

A relação entre os recursos do ambiente familiar mensurados pela RAF em dez questões e o desempenho cognitivo das crianças pode ser visualizada na Tabela 2 .

Observou-se que os passeios e viagens realizados pela criança, assim como a maior disponibilidade de brinquedos e materiais do infante (recursos do ambiente familiar) estão relacionados a melhores escores no teste de desenvolvimento cognitivo.

Os resultados da regressão logística binária podem ser visualizados na Tabela 3. No modelo de regressão linear binária hierárquica foram consideradas as variáveis que tiveram um $\mathrm{p}$ $<0,10$ na análise bivariada, a saber: - nível econômico, tipo de creche, escolaridade materna e paterna, passeios e viagens e recursos do ambiente familiar (brinquedos da criança). No modelo final permaneceram apenas aquelas com $\mathrm{p}<0,05$, a saber: - recursos do ambiente familiar (brinquedos da criança) e nível econômico.

As variáveis independentes recursos do ambiente familiar e nível econômico foram consideradas preditoras significativas para distinção entre crianças com desenvolvimento cognitivo abaixo e acima da média. Observa-se que crianças com mais recursos disponíveis no seu domicílio apresentaram 39,8 vezes mais chance de terem um melhor escore no teste de desenvolvimento cognitivo, assim como crianças de famílias classificadas com A, B e C1 pelo critério socioeconômico Brasil apresentaram 2,96 vezes mais chance de terem melhores resultados no teste cognitivo.

Tabela 1. Características socioeconômicas descritas segundo desenvolvimento cognitivo, 2017

\begin{tabular}{|c|c|c|c|c|c|c|c|}
\hline & & \multicolumn{4}{|c|}{ Desenvolvimento Cognitivo } & \multirow{3}{*}{$\begin{array}{c}\text { Teste } \\
\text { Qui-quadrado }\end{array}$} & \multirow{3}{*}{ Valor $\mathrm{p}$} \\
\hline & & \multicolumn{2}{|c|}{ Abaixo da média $(n=47)$} & \multicolumn{2}{|c|}{ Médio ou superior $(n=57)$} & & \\
\hline & & $\mathrm{n}$ & $\%$ & $\mathrm{n}$ & $\%$ & & \\
\hline \multirow{2}{*}{ Sexo } & Feminino & 20 & $42,6 \%$ & 31 & $54,4 \%$ & \multirow{2}{*}{1,44} & \multirow{2}{*}{0,230} \\
\hline & Masculino & 27 & $57,4 \%$ & 26 & $45,6 \%$ & & \\
\hline \multirow{2}{*}{ Nível econômico } & A, B, C1 & 14 & $29,8 \%$ & 36 & $63,2 \%$ & \multirow{2}{*}{11,49} & \multirow{2}{*}{$0,001^{*}$} \\
\hline & $\mathrm{C} 2, \mathrm{D}, \mathrm{E}$ & 33 & $70,2 \%$ & 21 & $36,8 \%$ & & \\
\hline \multirow{2}{*}{ Escolaridade materna } & Educação Básica & 35 & $83,3 \%$ & 31 & $54,4 \%$ & \multirow{2}{*}{9,11} & \multirow{2}{*}{$0,003^{*}$} \\
\hline & Educação superior & 07 & $16,7 \%$ & 26 & $45,6 \%$ & & \\
\hline \multirow{2}{*}{ Tipo de creche } & Particular & 5 & $10,6 \%$ & 24 & $42,1 \%$ & \multirow{2}{*}{12,68} & \multirow{2}{*}{$<0,001^{*}$} \\
\hline & Pública & 42 & $89,4 \%$ & 33 & $57,9 \%$ & & \\
\hline \multirow{2}{*}{ Escolaridade paterna } & Educação Básica & 37 & $92,5 \%$ & 34 & $64,2 \%$ & \multirow{2}{*}{10,1} & \multirow{2}{*}{$0,001^{*}$} \\
\hline & Educação superior & 03 & $7,5 \%$ & 19 & $35,8 \%$ & & \\
\hline
\end{tabular}

*Estatisticamente significativo

$\mathrm{n}=$ número de crianças 


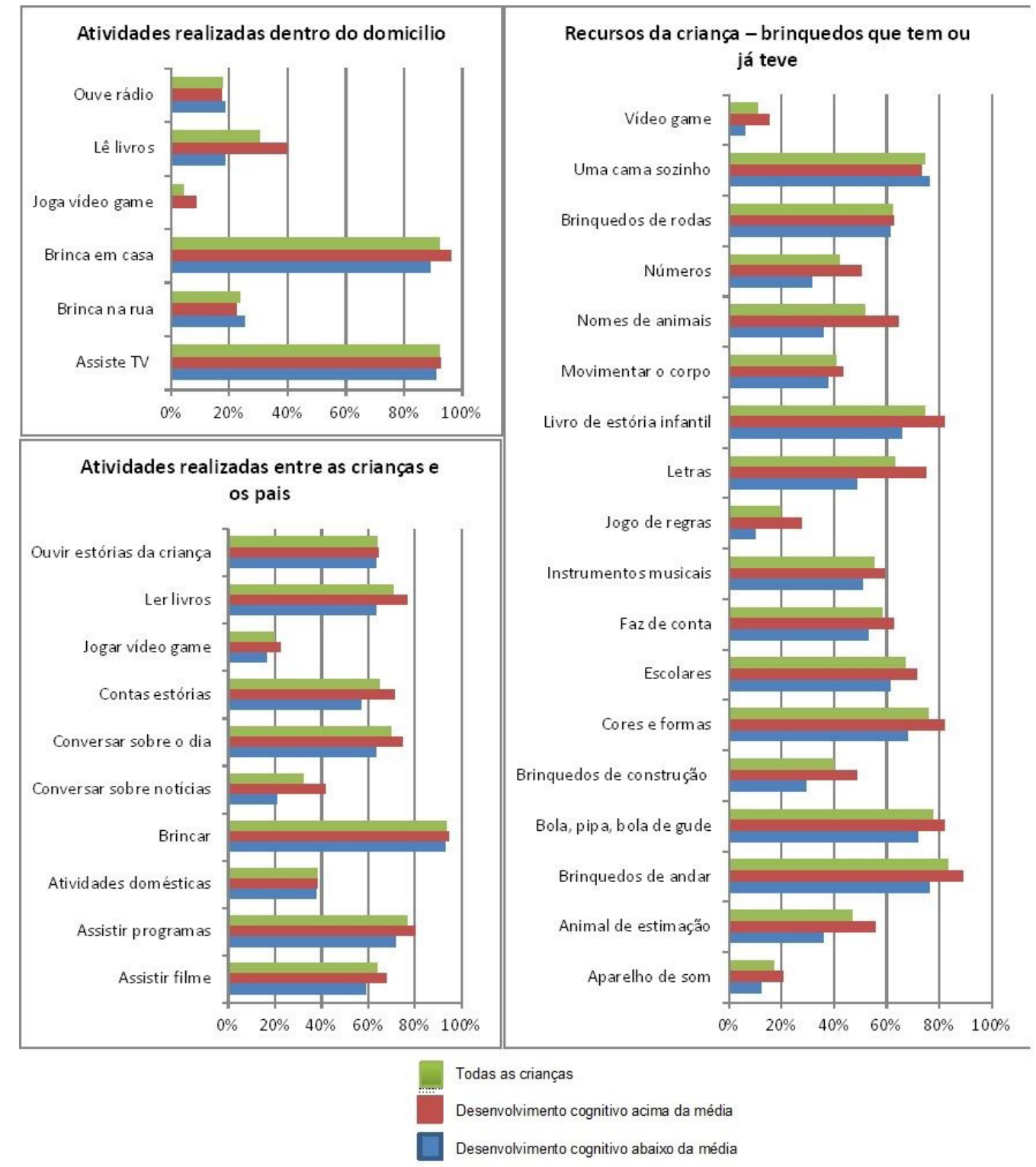

Legenda: $\mathrm{b}$ = coeficiente de regressão; $\mathrm{S} . \mathrm{E}$ = Margem de erro; $\mathrm{OR}=$ Razão de chance; IC= Intervalo de confiança

Figura 1. Características ambientais e práticas parentais

Tabela 2. Rotinas domiciliares

\begin{tabular}{|c|c|c|c|c|c|c|c|c|}
\hline & \multirow{3}{*}{$\begin{array}{c}\text { Escore } \\
\text { máximo } \\
\text { permitido }\end{array}$} & \multicolumn{6}{|c|}{ Desenvolvimento cognitivo } & \multirow{3}{*}{$\begin{array}{c}\text { Teste Mann- } \\
\text { Whitney } \\
\text { Valor } p\end{array}$} \\
\hline & & \multicolumn{3}{|c|}{ Abaixo da média $(n=47)$} & \multicolumn{3}{|c|}{ Médio ou superior $(n=57)$} & \\
\hline & & Mediana & $1^{\circ}$ Quartil & $3^{\circ}$ Quartil & Mediana & $1^{\circ}$ Quartil & $3^{\circ}$ Quartil & \\
\hline Atividades realizadas pela criança em casa & 6,00 & 3,0 & 2,0 & 3,5 & 2,0 & 2,0 & 3,0 & 0,154 \\
\hline Passeios e viagens & 19,00 & 3,0 & 2,0 & 5,0 & 5,0 & 3,0 & 7,0 & $0,016^{\star}$ \\
\hline Atividades programadas & 8,00 & 0,00 & 0,00 & 0,00 & 0,00 & 0,00 & 0,00 & 0,154 \\
\hline $\begin{array}{c}\text { Atividades dos pais com as crianças em } \\
\text { casa }\end{array}$ & 10,00 & 6,00 & 5,0 & 7,0 & 6,00 & 5,0 & 8,0 & 0,131 \\
\hline Brinquedos da criança & 18,00 & 8,0 & 6,0 & 12,0 & 11,00 & 8,0 & 14,0 & $0,003^{*}$ \\
\hline Conter jornais e revistas em casa & 7,00 & 1,00 & 0,00 & 1,00 & 1,00 & 0,00 & 1,00 & 0,367 \\
\hline Conter livros em casa & 7,00 & 3,00 & 2,0 & 4,0 & 3,00 & 2,0 & 5,0 & 0,364 \\
\hline $\begin{array}{l}\text { Acompanhamento da criança nos afazeres } \\
\text { da creche }\end{array}$ & 12,00 & 8,00 & 7,0 & 8,0 & 8,00 & 8,0 & 10,0 & 0,169 \\
\hline Horário certo para as atividades & 12,00 & 8,00 & 6,0 & 10,0 & 8,00 & 5,5 & 10,0 & 0,660 \\
\hline Reunião da família & 12,00 & 9.0 & 7,0 & 10,0 & 9,0 & 7,5 & 10,0 & 0,744 \\
\hline
\end{tabular}

${ }^{*}$ Estatisticamente significativo 
Tabela 3. Regressão logística binária hierárquica para variável dependente "desenvolvimento cognitivo"

\begin{tabular}{|c|c|c|c|c|}
\hline Variáveis independentes & $\mathrm{b} \pm \mathrm{S} . \mathrm{E}$ & OR & IC (95\%)a & $\mathrm{p}$ \\
\hline Recursos do ambiente familiar & $3,68 \pm 1,65$ & 39,86 & $1,54-1028,6$ & $0,026^{*}$ \\
\hline Nível econômico & $1,08 \pm 0,54$ & 2,96 & $1,02-8,63$ & $0,046^{\star}$ \\
\hline Passeios e viagens & $0,85 \pm 1,75$ & 0,43 & $0,14-13,79$ & 0,636 \\
\hline Escolaridade paterna & $0,17 \pm 0,69$ & 1,19 & $0,30-8,63$ & 0,800 \\
\hline
\end{tabular}

Qualidade do modelo segundo Hosmer e Lemeshow $=0,57$

${ }^{*} p=$ nível de significância $<0,05$

$a=$ Intervalo de confiança

\section{DISCUSSÃO}

Esta pesquisa buscou conhecer os fatores associados ao desenvolvimento cognitivo de crianças de 24 a 42 meses e observouse que há influência dos fatores estudados no desenvolvimento cognitivo. Considerando que as crianças participantes do presente estudo não possuem alterações estruturais e funcionais dos sistemas orgânicos, os fatores avaliados parecem ter influência direta nas experiências vivenciadas pelas crianças e refletir o desempenho delas nas tarefas cognitivas.

$\mathrm{Na}$ análise bivariada as variáveis: tipo de instituição educacional, escolaridade paterna, nível econômico, recursos no ambiente familiar tais como brinquedos de regras, lógica, números, de montar e passeios e viagens realizadas pela criança foram associados a um melhor desenvolvimento cognitivo, no entanto, no modelo final permaneceram apenas as variáveis recursos no ambiente familiar e nível econômico.

Neste estudo a maioria das crianças frequenta a rede pública de ensino. Revisão sistemática sobre a influência dos fatores ambientais no desenvolvimento infantil verificou prevalência de atraso em diferentes domínios do desenvolvimento e fatores de risco associados considerando crianças frequentadoras de creches públicas $^{(10)}$. Outro estudo comparou o desenvolvimento cognitivo e motor de crianças de mesmo nível socioeconômico, porém de instituições educacionais públicas e privadas, e encontrou pior desempenho em todos os domínios do desenvolvimento nas crianças das creches públicas ${ }^{(19)}$. Possível justificativa está no fato de que creches particulares apresentam melhor estrutura física, brinquedos e atividades mais adequadas que creches públicas segundo avaliações do sistema educacional brasileiro $^{(24)}$. No presente estudo, a variável tipo de creche não se manteve associada ao desenvolvimento cognitivo no modelo final. No entanto, torna-se necessário reforçar a importância do investimento na educação pública, visto que quem as frequenta já parece dispor de menos recursos ambientais e parentais para um melhor desenvolvimento. Ademais, o investimento em politicas e programas em prol do desenvolvimento infantil é uma das metas da OMS em níveis local, nacional e global ${ }^{(3)}$.

A baixa escolaridade materna e paterna foram fatores associados ao pior desenvolvimento, sendo que das crianças com desenvolvimento abaixo da média, o pai e a mãe possuíam, em $92,5 \%$ e $83,3 \%$ delas, respectivamente, somente educação básica. Pais e mães com níveis educacionais mais altos tem oportunidades de melhores empregos, tendem a fornecer uma melhor qualidade do ambiente familiar geral, com maior oferta de materiais e brinquedos que incentivem o aprender, proporcionando maior enriquecimento e variedade de experiências para a criança ${ }^{(10)}$. Um estudo mostra que a qualidade do ambiente que a criança está inserida, e a quantidade de livros presente no domicílio foram positivamente previsto pela escolaridade paterna e materna ${ }^{(25)}$. Embora a escolaridade parental não tenha permanecido no modelo final do presente estudo como fator preditor do desenvolvimento cognitivo das crianças, ela está diretamente associada ao nível econômico da família, sendo um dos itens considerados para a classificação do nível econômico ${ }^{(21)}$.

As opções para brincar e o tipo de brinquedo que a criança possui, de acordo com os resultados, também influenciam o desenvolvimento cognitivo. Notou-se diferença estatística entre as crianças com desenvolvimento cognitivo acima e abaixo da média quanto à posse de brinquedos com letras $(p=0,011)$, brinquedos com nomes de animais $(p=0,006)$ e jogos de regras $(p=0,035)$. Estudo sobre desenvolvimento da comunicação de crianças de um a três anos e sua relação com o ambiente familiar revelou associação positiva entre os brinquedos que a criança tem em casa e o melhor perfil comunicativo ${ }^{(14)}$. O desenvolvimento da linguagem, da lógica e a capacidade de concentração são mais aguçados nas crianças que utilizam brinquedos envolvendo números e charadas, os quais exigem raciocínio lógico, pensamento abstrato e a rapidez de raciocínio para a efetivação das brincadeiras ${ }^{(26)}$. Em contrapartida um estudo relacionou a quantidade de brinquedos presentes no ambiente com a qualidade da brincadeira. Notou-se que quanto menos brinquedos disponíveis, as crianças engajam em períodos mais longos, permitindo explorar mais o objeto de uma forma mais criativa ${ }^{(17)}$. Possível justificativa está no fato de famílias que não dispõem de recursos para oferecer grande quantidade de brinquedos, poderem garantir qualidade no brincar a seus filhos, reforçando o importante papel da família na mediação dos efeitos negativos da exposição da criança em ambiente com baixo nível socioeconômico ${ }^{(5,6)}$.

$\mathrm{O}$ ato de brincar possibilita o processo de aprendizagem da criança, pois facilita a construção da reflexão, da autonomia e da criatividade, sendo crucial para o desenvolvimento de capacidades importantes como a atenção, a memória, a imitação, a imaginação ${ }^{(27)}$. É importante ressaltar que no contexto de famílias economicamente desfavorecidas há redução dos recursos e materiais de aprendizagem, os quais influenciam diretamente o desenvolvimento da criança $\mathrm{a}^{(9)}$.

Ao comparar as crianças dos dois grupos, com desenvolvimento abaixo da média com as do grupo médio ou superior, observou-se associação positiva dos passeios e viagens realizadas pela criança no último ano $(\mathrm{p}=0,016)$ com o melhor desenvolvimento cognitivo. A realização de passeios específicos e em diferentes lugares nos últimos doze meses $(71,6 \%)$ tal como: bosque, circo, 
cinema ou teatro, parque de diversões, praia foi associada ao melhor desempenho acadêmico de escolares de uma metrópole brasileira $^{(28)}$. Ao realizar um passeio, a criança experimenta diversos tipos de estímulos diferentes, conhece novas culturas e convive com pessoas que não estão presentes no seu dia-a-dia, concedendo a oportunidade de riqueza e variedade de estímulos, os quais determinam suas perspectivas futuras e desenvolvimento de novas habilidades ${ }^{(8)}$. A instituição de ensino de qualidade, por sua vez, aparece como mediadora do risco psicossocial, sendo capaz de oportunizar a crianças experiências potencializadoras do desenvolvimento, as quais podem minimizar as carências provenientes das condições econômicas desfavorecidas e de um ambiente familiar desprovido de recursos ${ }^{(24)}$.

$\mathrm{Na}$ análise multivariada os recursos do ambiente familiar e o nível econômico que a criança está inserida permaneceram como principais fatores associados ao desenvolvimento cognitivo. Observou-se que crianças de famílias classificadas com A, B e C1 pelo Critério Econômico Brasil apresentaram 2,96 vezes mais chance de terem melhores resultados no teste cognitivo quando comparadas com famílias classificadas como C2, D ou E. A baixa qualidade do ambiente de casa pode estar associada à baixa renda mensal e menor variedade de recursos e estímulos com privação de novas oportunidades ao infante ${ }^{(3,10)}$. A pobreza infantil foi relatada como mais fortemente associada a piores índices de desenvolvimento cognitivo e a dificuldades de aprendizagem escolar no decorer do tempo ${ }^{(9)}$. Um estudo verificou que a renda familiar, além de variáveis demográficas, se associou com suspeitas de atraso no desenvolvimento de 176 crianças com desenvolvimento típico, na faixa etária de $24 \operatorname{meses}^{(29)}$. A taxa de pobreza infantil é, portanto, um indicador-chave da saúde e bem-estar de uma sociedade, sendo um importante índice que determina como renda e privação na infância comprometem o desenvolvimento saudável das crianças e surgimento de novas oportunidades $^{(9)}$.

No presente estudo crianças com mais recursos disponíveis no seu domicílio apresentaram 39,8 vezes mais chance de terem um melhor escore no teste de desenvolvimento cognitivo. Tais dados corroboram com um estudo realizado com 350 crianças na faixa etária de 17 a 42 meses, onde observaram que em uma regressão linear univariada o aumento de um ponto na escala do inventário HOME implicou num incremento de 0,659 no desempenho cognitivo ${ }^{(12)}$. $\mathrm{O}$ ambiente físico em que a criança convive, seja no contexto familiar ou escolar, quando organizado e com possibilidades de interação, se torna um fator de resiliência diante das adversidades físicas ou psicológicas ${ }^{(30)}$. Para se tornar promotor, o ambiente necessita dar variedades de estímulos e oportunidades à criança, em concordância com os achados desta pesquisa.

O presente estudo apresenta como limitações o fato de os recursos do ambiente familiar serem investigados sob entrevista semi-estruturada, sujeitos ao viés de memória e desejabilidade social.

Os achados sugerem que os recursos do ambiente familiar e os tipos de brinquedos que a criança possui podem ser fatores mais importantes a serem considerados do que simplesmente a instituição de ensino que a criança frequenta, o que reforça a importância da família como alicerce do desenvolvimento infantil.
Nesse sentido, o presente estudo vai ao encontro de uma demanda atual no cuidado da população pediátrica, no que se refere à constituição de indicadores que atribuam a qualidade ao desenvolvimento da criança. A escolaridade dos pais ${ }^{(10),} \mathrm{o}$ nível econômico da família ${ }^{(3)}$, os recursos presentes no ambiente familiar ${ }^{(9,10)}$, determinam oportunidades de interação das crianças com outras pessoas, especialmente os pais, e de vivências estimuladoras do desenvolvimento cognitivo, reforçando a importância de um ambiente rico em recursos físicos e humanos na potencialização das habilidades da criança.

\section{CONCLUSÃO}

Ao ser investigados fatores como: tipo de instituição educacional, escolaridade paterna, nível econômico, recursos no ambiente familiar e passeios e viagens realizadas pela criança, apenas as variáveis recursos no ambiente familiar e nível econômico foram associados a um melhor desenvolvimento cognitivo.

Os recursos do ambiente familiar e os tipos de brinquedos que a criança possui podem ser fatores mais importantes a serem considerados do que simplesmente a instituição de ensino que a criança frequenta, o que reforça a importância da família como alicerce do desenvolvimento infantil.

É necessário traçar estratégias destinadas a refletir sobre atuação da família e da escola nos primeiros anos de vida, especialmente nos casos de maior vulnerabilidade socioeconômica, a fim de minimizar as desigualdades e sua influência nas oportunidades futuras dessas crianças.

\section{REFERÊNCIAS}

1. Mussen PH, Conger JJ, Kagan J, Huston AC. Desenvolvimento e personalidade da criança. São Paulo: Editora Harbra; 1977.

2. Halpern R, Giugliani ER, Victora CG, Barros FC, Horta BL. Risk factors for suspicion of developmental delays at 12 months of age. J Pediatr. 2000;76(6):421-8. http://dx.doi.org/10.2223/JPED.88. PMid:14647629.

3. Black MM, Walker SP, Fernald LCH, Andersen CT, DiGirolamo AM, Lu $\mathrm{C}$, et al. Early childhood development coming of age: science through the life course. The Lanced. 2017;389(10064):77-90. http://dx.doi.org/10.1016/ S0140-6736(16)31389-7. PMid:27717614.

4. OMS: Organização Mundial da Saúde. Criança e adolescente: o que fazer para cuidar. Geneva: OMS; 2018.

5. FMCSV: Fundação Maria Cecília Souto Vidigal. Primeiríssima infância: creche: necessidades e interesses das famílias e crianças. São Paulo: Fundação Maria Cecília Souto Vidigal; 2017.

6. Black MM, Walker SP, Fernald LCH, Andersen CT, DiGirolamo AM, Lu C, et al. Early childhood development coming of age: science through the life course. Lancet. 2017;389(10064):77-90. http://dx.doi.org/10.1016/ S0140-6736(16)31389-7. PMid:27717614.

7. Halpern R. Manual de pediatria do desenvolvimento e comportamento. 1. ed. São Paulo: Manole; 2015.

8. Lee G, McCreary L, Kim MJ, Park CG, Yang S. Individual and environmental factors influencing questionable development among low-income children: differential impact during infancy versus early childhood. J Korean Acad Nurs. 2012;42(7):1039-49. http://dx.doi.org/10.4040/jkan.2012.42.7.1039. PMid:23377600.

9. Chaudry A, Wimer C. Poverty is not just an indicator: the relationship between income, poverty, and child well-being. Acad Pediatr. 2016;16(3, Supl):S23-9. http://dx.doi.org/10.1016/j.acap.2015.12.010. PMid:27044698. 
10. Morais RLS, Carvalho AM, Magalhães LC. O contexto ambiental e o desenvolvimento na primeira infância: estudos brasileiros. J Phys Educ. 2016;27:e2714. http://dx.doi.org/10.4025/jphyseduc.v27i1.2714.

11. Ferriolli SHT, Marturano EM, Puntel LP. Contexto familiar e problemas de saúde mental infantil no Programa Saúde da Família. Rev Saude Publica. 2007;41(2):251-9. http://dx.doi.org/10.1590/S0034-89102006005000017. PMid:17384801.

12. Andrade SA, Santos DN, Bastos AC, Pedromônico MRM, Almeida-Filho N, Barreto ML. Family environment and child's cognitive development: an epidemiological approach. Rev Saude Publica. 2005;39(4):606-11. http:// dx.doi.org/10.1590/S0034-89102005000400014. PMid:16113911.

13. Guimarães AF, Carvalho DV, Machado N, Baptista RA, Lemos SM. Risk of developmental delay of children aged between two and 24 months and its association with the quality of family stimulus. Rev Paul Pediatr. 2013;31(4):452-8. http://dx.doi.org/10.1590/S0103-05822013000400006. PMid:24473949.

14. Dourado JS, Carvalho SAS, Lemos SMA. Development of communication of children aged between one and three years old and their relationship with the family and school environments. Rev CEFAC. 2015;17(1):88-99. http://dx.doi.org/10.1590/1982-0216201515013.

15. Marturano EM. The home environment resources scale. Psicol Reflex Crit. 2006;19(3):498-506. http://dx.doi.org/10.1590/S0102-79722006000300019.

16. Chang JH, Yeh TL. The influence of parent-child toys and time of playing together on attachment. Proced Manufactu. 2015;3:4921-6. http://dx.doi. org/10.1016/j.promfg.2015.07.628.

17. Dauch C, Imwalle M, Ocasio B, Metz AE. The influence of the number of toys in the environment on toddlers' play. Infant Behav Dev. 2018;50:7887. http://dx.doi.org/10.1016/j.infbeh.2017.11.005. PMid:29190457.

18. Neves KR, Morais RLS, Teixeira RA, Romero AT, Pinto PAF. Crescimento e desenvolvimento e seus determinantes ambientais e biológicos. J Pediatr. 2016;92(3):241-50. PMid:26772895.

19. Santos MM, Corsi C, Marques LAP, Rocha NACF. Comparison of motor and cognitive perfomance of children attending public and private day care centers. Braz J Phys Ther. 2013;17(6):579-87. http://dx.doi.org/10.1590/ S1413-35552012005000126. PMid:24346293.

20. Bayley N. Bayley scales of infant and and toddller development: technical manual. 3rd ed. San Antonio: Pearson; 2006.

21. ABEP: Associação Brasileira de Empresas de Pesquisa. Critério de Classificação Econômica Brasil. São Paulo: ABEP; 2015.
22. Souza NT, Chagas PSC, Campos GG, Valenzuela EJ, Frônio JS, Ribeiro LC. Functionality of children aged 5 to 7 years born prematurely. Fisioter Mov. 2017;30(Supl 1):S121-9. http://dx.doi.org/10.1590/1980-5918.030. s01.ao12.

23. Brasil. Lei ${ }^{\circ}$ 9.394, de 20 de dezembro de 1996. Estabelece as diretrizes e bases da educação nacional. Diário Oficial da União; Brasília; 23 dez. 1996.

24. Oliveira MA, Furtado RA, Souza TN, Carvalho MIC. Avaliação de ambientes educacionais infantis. Paidéia. 2003;13(25):41-58.

25. Jeong J, McCoy DC, Fink G. Pathways between paternal and maternal education, caregivers' support for learning, and early child development in 44 low-and middle-income countries. Early Child Res Q. 2017;41:136-48. http://dx.doi.org/10.1016/j.ecresq.2017.07.001.

26. Hansen J, Macarini SM, Martins GDF, Wanderlind FH, Vieira ML. The play behavior and its implications for child development according to evolutionary psychology. Rev Bras Crescimento Desenvolv Hum. 2007;17(2):133-43.

27. Fantacholi FN. O brincar na educação infantil: jogos, brinquedos e brincadeiras: um olhar psicopedagógico. Rev Cien Aprender. 2011;5.

28. Osti A. The familiar context and the performance of 5th grade students from a school in the inner region of Sao Paulo. ETD - Educ Temat Digit. 2016;18(2):369-83.

29. Lamy F Fo, Medeiros SM, Lamy ZC, Moreira MEL. Ambiente domiciliar e alterações do desenvolvimento em crianças de comunidade da periferia de São Luís - MA. Cien Saude Colet. 2011;16(10):4181-7. http://dx.doi. org/10.1590/S1413-81232011001100023.

30. Alves JMM, Carvalho AJA, Pereira SCG, Escarce AG, Goulart LMHF, Lemos SMA. Associação entre desenvolvimento de linguagem e ambiente escolar em crianças da educação infantil. Distúrb Comun. 2017;29(2):34253. http://dx.doi.org/10.23925/2176-2724.2017v29i2p342-353.

\section{Contribuição dos autores}

LP participou da análise de dados, redação e elaboração do artigo; SCG participou da revisão do conteúdo do manuscrito e aprovação da versão final; RLSM participou da concepção, planejamento e coorientação do projeto; JNPN participou do planejamento do projeto e revisão do conteúdo do manuscrito; JNS realizou a orientação do trabalho, análise e interpretação de dados estatísticos e revisão do manuscrito. 